\title{
Thrombus in a normal sinus of Valsalva: angiographic, multiplane transoesophageal echocardiographic, and surgical findings
}

\author{
Luc Christiaens, Joseph Allal, Pierre Corbi
}

Department of Cardiology A, Hospital University, Poitiers,

France

L Christiaens

J Allal

Department of

Thoracic and

Cardiovascular

Surgery, Hospital

University, Poitiers,

Prance

Correspondence to:

Dr L Christiaens, Service de Cardiologie A, Centre Hospitalo-universitaire de Poitiers, 350 avenue Jacques Coeur BP 577, 86021 Poitiers, France.

Accepted for publication 23 May 1996

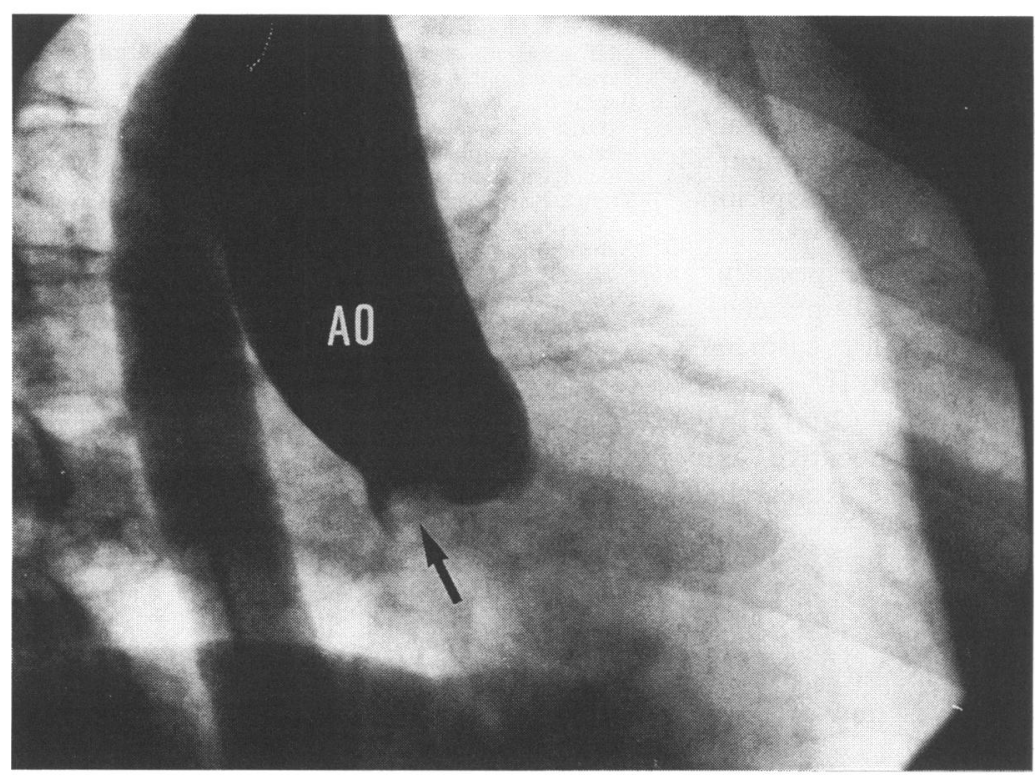

Figure 1 Aortogram, in the right anterior oblique projection, showing the ascending aorta (AO) and the non-opacification of the non-coronary sinus of Valsalva corresponding to the thrombus (arrow).

\begin{abstract}
A large intraluminal thrombus within an otherwise normal sinus of Valsalva was diagnosed in a 41 year old man who was investigated for myocardial infarction. The thrombus was suspected by aortic root injection, confirmed by transoesophageal echocardiography, and treated surgically.
\end{abstract}

(Heart 1996;76:287-288)

Keywords: thrombus; sinus of Valsalva; aorta

The advent of transoesophageal echocardiography has made it possible to detect during life atherosclerotic plaques protruding into the aortic arch and descending aorta ${ }^{12}$ but pure thrombus in the thoracic aorta has rarely been seen. Appropriate treatment of patients with documented thrombi in the aorta has not been defined. We describe a patient with a thrombus within a normal sinus of Valsalva and the angiographic and multiplane transoesophageal echocardiographic findings when thrombus is present at this very unusual site.

\section{Case report}

A 41 year old man was admitted with an acute myocardial infarction of the inferior wall in April 1995. This hypertensive patient had sustained an ischaemic right cerebellar vascular accident in 1988 from which he recovered fully. In 1994 an acute inferior wall myocardial infarction developed and he had elective angioplasty of the right coronary artery. The exercise stress test was normal in September 1994.

In April 1995 he was referred to our hospital 24 hours after another inferior wall myocardial infarction. He was treated medically with intravenous heparin (180 UI/kg/day), infusion of nitrates, oral aspirin ( $250 \mathrm{mg}$ daily), and atenolol (200 mg daily). Cardiac catheterisation, performed five days later, showed an occlusion of the right coronary artery and of the left circumflex artery just after the posterolateral branch. Five days later a second coronary angiogram was performed because unstable angina recurred. This confirmed the occlusion of the right coronary artery but showed extension of the thrombus with occlusion of the proximal part of the left circumflex artery and of the posterolateral branch.

There was also no opacification of the noncoronary sinus of Valsalva on the thoracic aortogram in the right anterior oblique projection (fig 1). For a more comprehensive assessment of the aortogram multiplane transoesophageal echocardiography performed immediately (Vingmed Sonotron CFM 750, $5 \mathrm{MHz}$ transoesophageal probe). The echocardiographic examination clearly showed a round motionless mass (diameter $=10 \mathrm{~mm}$ ) filling the noncoronary sinus of Valsalva, without aneurysm of the sinus, aortic intimal flap, or aortic valve abnormality (figs 2 and 3). A large and mobile thrombus was suspected and this diagnosis was confirmed by surgery and pathological examination. No aortic wall injury or atheromatous lesions was noted during surgical exploration and a saphenous vein graft was anastomosed to the posterolateral coronary artery. There were no cardiac complications but 48 hours later there was critical ischaemia of the right leg with a thrombus in the right femoral artery. This was successfully treated by surgical thrombectomy. This patient required another surgical thrombectomy 23 days later for similar ischaemic damage of the 


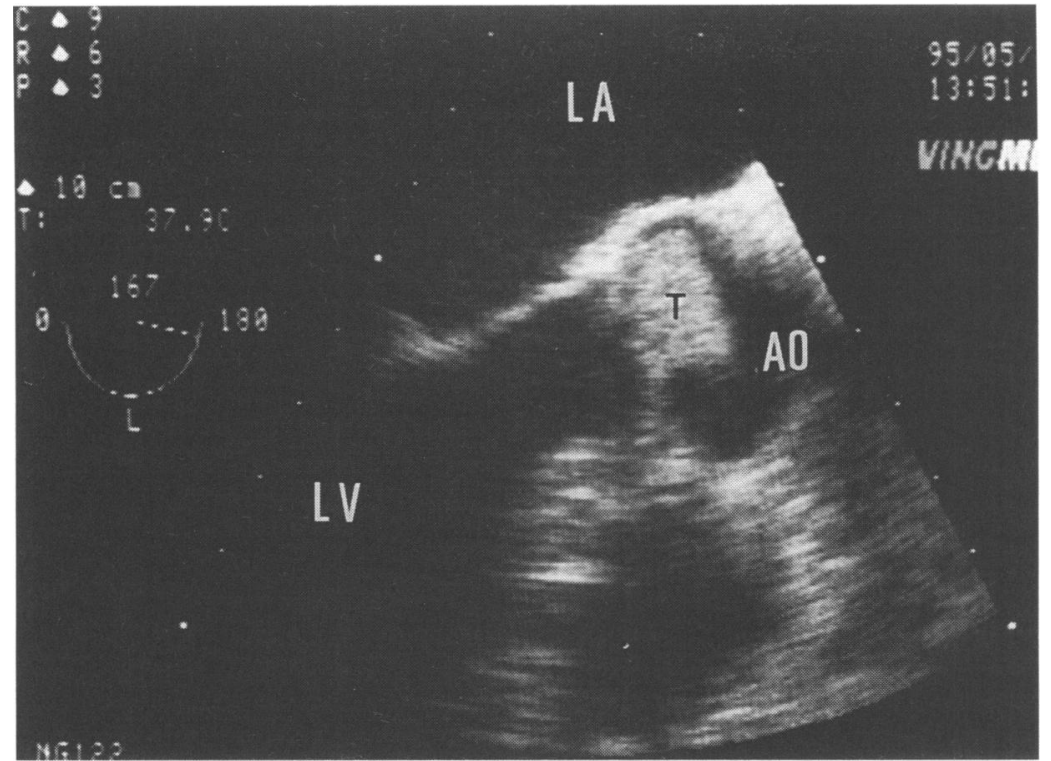

Figure 2 Multiplane transoesophageal echocardiography, long axis section at $167^{\circ}$ rotation, in diastole: thrombus $(T)$ just behind the non-coronary cusp. $A O$, aorta; $L A$, left atrium; $L V$, left ventricle.

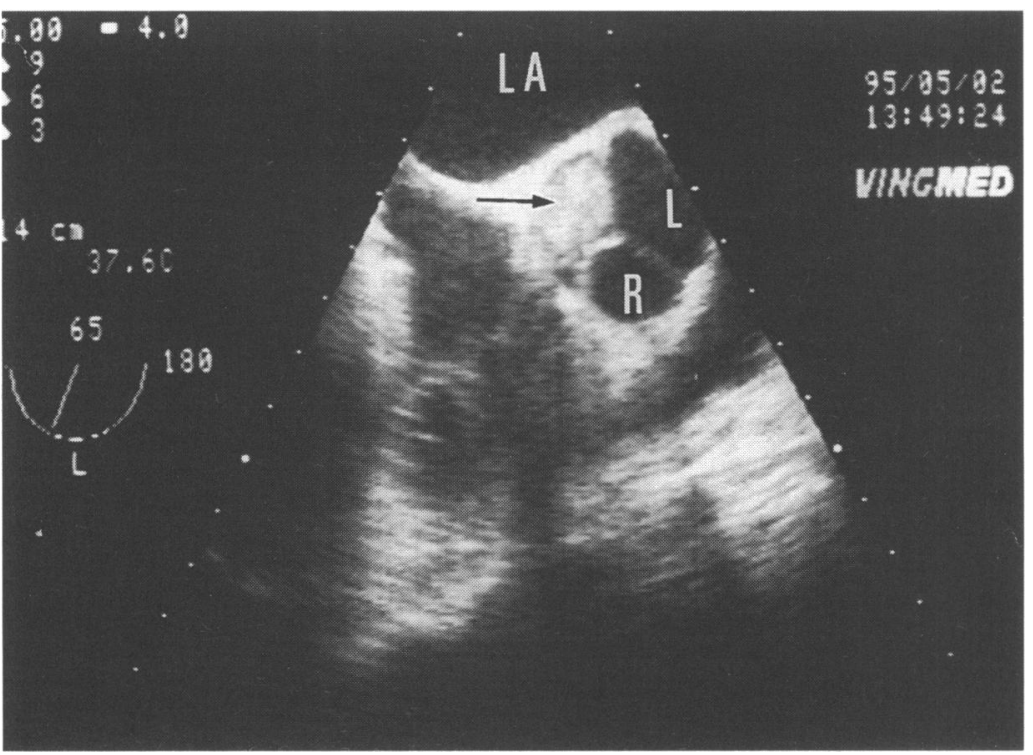

Figure 3 Multiplane transoesophageal echocardiography, oblique short axis view of the aortic valve in diastole, showing thrombus filling the non-coronary sinus of Valsalva (arrow). $L$, left coronary cusp; $R$, right coronary cusp; $L A$, left atrium.

right leg during an accidental decrease of oral anticoagulation. Follow up during treatment with oral anticoagulation, atenolol, and aspirin was uneventful.

Laboratory data were normal (especially protein $\mathrm{C}$ and $\mathrm{S}$, antithrombin III, platelet count, and haematocrit) except for cardiac enzymes (peak creatine kinase (2450 IU)), fibrinogen $(9 \mathrm{~g} / \mathrm{l})$, total cholesterol $(2.66 \mathrm{~g} / \mathrm{l})$. Anticardiolipin antibodies were negative as was the VDRL (Venereal Disease Research Laboratories) serological test.

\section{Discussion}

The technology of multiplane transoesophageal echocardiography is now widely available and has proved extremely useful in evaluating cardiovascular anatomy, cardiac sources of embolisation, and atherosclerotic lesions in the thoracic aorta. ${ }^{3}$ Transoesophageal echocardiography is ideally suited to evaluation of the proximal part of the thoracic root and the sinus of Valsalva. In the current case multiplane transoesophageal echocardiography clearly showed a thrombus in the non-coronary sinus of Valsalva without atherosclerotic lesions or aneurysm. Other published reports describe thromboembolic events complicating a large aneurysm of a sinus of Valsalva, ${ }^{45}$ thrombi related to plaques in the thoracic aortic, ${ }^{6-9}$ or mobile thrombus in the proximal part of the descending thoracic aorta. ${ }^{89}$ Our patient had no local aortic wall lesion secondary to the catheterisation and no antithrombin III deficiency or antiphospholipid syndrome, unlike previously reported cases. ${ }^{111}$ To date, only three patients with aortic thrombectomy have had a successful outcome $^{78}$ and there is one report of resolution of a large aortic thrombus after thrombolytic therapy with thrombolysis. ${ }^{12}$ Systemic anticoagulation remains the mainstay of treatment in aortic thrombosis. ${ }^{8}$ In our case, because the thrombus occurred despite anticoagulation and was associated with myocardial ischaemia and high risk of emboli, surgery was recommended. To our knowledge this is the first angiographic and transoesophageal echocardiographic description of a thrombus within an otherwise normal sinus of Valsalva.

1 Tunick PA, Rosenzweig BP, Katz ES, Freedberg RS, Perez $\mathrm{JL}$, Krozon I. High risk for vascular events in patients with protruding aortic atheromas: a prospective study. $f$ Am Coll Cardiol 1994;23:1085-90.

2 Amarenco P, Cohen A, Tzourio C, Bertrand B, Hommel M, Besson G, et al. Atherosclerotic disease of the aortic arch and the risk of ischemic stroke. N Engl f Med 1994; 331:1474-9.

3 Seward JB, Khandheira BK, Freeman WK, Oh JK, Enriquez-Sarano M, Miller FA, et al. Multiplane transesophageal echocardiography: image orientation, examination technique, anatomic correlations, and clinical applications. Mayo Clin Proc 1993;68:523-51.

4 Shahrabani RM, Jairaj PS. Unruptured aneurysm of the sinus of Valsalva: a potential source of cerebrovascular sinus of Valsalva: a potential source

5 Reid PG, Goudevenos JA, Hilton CJ. Thrombosed saccular aneurysm of a sinus of Valsalva: unusual cause of a lar aneurysm of a sinus of Valsalva: unusual
mediastinal mass. Br Heart $f$ 1990;63:183-5.

6 Bansal RC, Pauls GP, Shankel SW. Blue digit syndrome: transesophageal echocardiographic identification of thoracic aortic plaque-related thrombi and successful outcome with warfarin. Echocardiography 1993;6:319-23.

7 Tunick PA, Lackner H, Katz ES, Culliford AT, Giangola G, Kronzon I. Multiple emboli from a large aortic arch thrombus in a patient with thrombotic diathesis. Am Heart $\mathcal{F} 1992 ; 124: 239-41$.

8 Dee W, Geibel A, Kasper W, Konstantinides S, Just H. Mobile atherosclerotic lesions of the thoracic aorta: the diagnostic impact of transesophageal echocardiography. Am Heart f 1993;126:707-9.

9 Freedberg RS, Tunick PA, Culliford AT, Tatelbaum RJ, Kronzon I. Disappearance of a large intraortic mass in a patient with prior systemic embolization. Am Heart $f$ 1993;125:1445-7.

10 Shapiro ME, Rodvien R, Bauer KA, Salzmann EW. Acute aortic thrombosis in antithrombin-III deficiency. $\mathcal{F} A M A$ 1981;245:1759-61.

11 Bruce D, Bateman D, Thomas $R$. Left ventricular thrombi in a patient with the antiphospholipid syndrome. $\mathrm{Br}$ Heart f 1995;74:202-3.

12 Hausmann D, Gulba D, Bargheer $\mathrm{K}$, Niedermeyer J, Comess KA, Daniel WG. Successful thrombolysis of an aortic-arch thrombus in a patient after mesenteric embolism. N Engl f Med 1992;327:500-1. 